\title{
Prognostic Value of Rapid Shallow Breathing Index for Weaning Success in Intensive Care Unit Patients under Mechanical Ventilation
}

\author{
Md. Sirajul Islam¹, Md. Ali Haider², Uzzwal Kumar Mallick³, Mohammad Asaduzzaman, \\ Md. Gias Uddin ${ }^{5}$, Kazi Eshika Raka ${ }^{6}$, Farhana Mamtaz', Syed Iftekhar Ahmed ${ }^{8}$, \\ Dewan Mohammad Kudrat-A-Elahi ${ }^{9}$, Khurshida Samad ${ }^{10}$, Md. Abdus Salam ${ }^{11}$
}

${ }^{1}$ Assistant Professor, Department of Critical Care Medicine, National Institute of Neurosciences \& Hospital, Dhaka, Bangladesh; ${ }^{2}$ Assistant Professor, Department of Anesthesia, Green Life Medical College, Dhaka, Bangladesh; ${ }^{3}$ Registrar, Department of Critical Care Medicine, National Institute of Neurosciences \& Hospital, Dhaka, Bangladesh; ${ }^{4}$ Assistant Registrar, Department of Critical Care

Medicine, National Institute of Neurosciences \& Hospital, Dhaka, Bangladesh; ${ }^{5}$ Post Graduate Student (Phase B Residence,

Anesthesiology), Department of Anesthesia, Pain, Palliative \& Intensive Care Unit, Dhaka Medical College \& Hospital, Dhaka,

Bangladesh; ${ }^{6}$ Medical Officer, Department of Critical Care Medicine, National Institute of Neurosciences and Hospital,

Dhaka, Bangladesh; ${ }^{7}$ Medical Officer, Department of Critical Care Medicine, National Institute of Neurosciences and Hospital, Dhaka, Bangladesh; ${ }^{8}$ Medical Officer, Department of Critical Care Medicine, National Institute of

Neurosciences and Hospital, Dhaka, Bangladesh; ${ }^{9}$ Medical Officer, Department of Critical Care Medicine, National Institute of Neurosciences \& Hospital, Dhaka, Bangladesh; ${ }^{10}$ Assistant Professor, Department of Pathology,

National Institute of Ophthalmology, Dhaka, Bangladesh; 11Associate Professor, Department of Neurotrauma,

National Institute of Neurosciences \& Hospital, Dhaka, Bangladesh

[Received: 1 October 2019; Accepted: 12 November 2019; Published: 1 January 2020]

\begin{abstract}
Background: The weaning success in intensive care unit patients under mechanical ventilation is very important. Objective: The purpose of this study was to investigate the efficacy and effects of rapid shallow breathing index (RSBI) in predicting weaning success in patients with prolonged mechanical ventilation more than 48 hours. Methodology: This prospective cohort study was conducted in the Department of Anesthesia, Pain Palliative \& Intensive Care Unit of Dhaka Medical College Hospital, Dhaka, Bangladesh from January 2014 to December 2015 for a period of two (02) years. Patients on mechanical ventilation more than 48 hours with the age of 18 to 60 years were included in this study. During the weaning process, the arterial blood gases (ABG) values was checked and the patients was separated from mechanical ventilation. After measuring RSBI, patients was separated from mechanical ventilator and given T-piece trial (1 to 4 hours) and finally extubated as per advice of ICU consultant and observed for 48 hours. The patients were divided in two groups low RSBI $\leq 105$ breath/min/L and high RSBI $>105$ breath/min/L. These patients were prospectively followed up to 48 hours in ICU and HDU. Result: A total of 117 patients were included in this study. The validity of RSBI evaluation for trail failure was correlated by calculating sensitivity, specificity, accuracy, positive and negative predictive values. The sensitivity of RSBI was 54.5\% (95\% CI $23.38 \%$ to $83.25 \%)$ and specificity was $82.1 \%(95 \%$ CI $73.43 \%$ to $88.85 \%)$. However, positive predictive value and negative predictive value were $24.0 \%$ (95\% CI $13.84 \%$ to $38.30 \%$ ) and $94.6 \%$ (95\% CI $90.05 \%$ to $97.10 \%$ ) respectively. The accuracy was found $79.5 \%$ (95\% CI $71.03 \%$ to $86.39 \%)$. Receiver-operator characteristic (ROC) were constructed using RSBI of the weaning outcome, which gave a RSBI cut off value of $\geq 88$ as the value with a best combination of sensitivity (72.7\%) and specificity $(61.3 \%)$, accuracy $(60.7 \%)$, positive predictive value $(15.7 \%)$, negative predictive value $(95.5 \%)$ for trail failure. Conclusion: In conclusion the efficacy and effects of rapid shallow breathing index is found low sensitivity with high specificity in predicting weaning success in patients with prolonged mechanical ventilation more than 48 hours. [Journal of National Institute of Neurosciences Bangladesh, 2020;6(1): 9-14]
\end{abstract}

Keywords: : Prognostic value; rapid shallow breathing index; weaning success; intensive care unit; patients; mechanical ventilation

Correspondence: Dr. Md. Sirajul Islam, Assistant Professor, Department of Critical Care Medicine, National Institute of Neurosciences \& Hospital, Sher-E Bangla Nagor, Dhaka, Bangladesh; Email: sirajulrpmc@gmail.com; Cell no.: 8801712818254 Conflict of interest: There is no financial conflict of interest relevant to this paper to disclose.

Funding agency: This research project was not funded by any group or any institution.

Contribution to authors: Islam MS, Haider MA, Mallick UK have contributed from the protocol preparation, data collection up to report writing. Manuscript writing has been performed by Asaduzzaman M, Uddin MG, Raka KE, Mamtaz F. Ahmed SI, Kudrat-A-Elahi DM, Samad K, Salam MA have revised the manuscript.

How to cite this article: Islam MS, Haider MA, Mallick UK, Asaduzzaman M, Uddin MG, Raka KE, Mamtaz F, Ahmed SI, Kudrat-A-Elahi DM, Samad K, Salam MA. Prognostic Value of Rapid Shallow Breathing Index for Weaning Success in Intensive Care Unit Patients under Mechanical Ventilation. J Natl Inst Neurosci Bangladesh, 2020;6(1): 9-14

Copyright: (C2020. Islam et al. Published by Journal of National Institute of Neurosciences Bangladesh. This article is published under the Creative Commons CC BY-NC License (https://creativecommons.org/licenses/by-nc/4.0/). This license permits use, distribution and reproduction in any medium, provided the original work is properly cited, and is not used for commercial purposes. 


\section{Introduction}

Endotracheal intubation and mechanical ventilation (MV) are two separate, distinct processes that occur together in critical care unit in order to be useful ${ }^{1}$. Endotracheal intubation is the insertion of a specialized tube into the trachea, for the purpose of maintaining a patent airway, managing overwhelming secretion and providing oxygenation or mechanical ventilation. Mechanical ventilation is the process of providing ventilation with a ventilator in order to maintain respiratory function ${ }^{2}$. Endotracheal intubation and mechanical ventilation are the most frequently performed and most costly interventions in intensive care units to support the respiratory function ${ }^{3}$.

Mechanical ventilation is an invasive procedure and is associated with many serious complications, adverse physiological and psychological experiences ${ }^{4}$. The complications include injury to the vocal cords, trachea or larynx, tracheal stenosis, haemoptysis, ventilator-associated pneumonia (VAP), increased need for sedation, increased gastro-intestinal stress, skin breakdown and decubitus ulcers, muscle wasting, muscle weakness and pulmonary barotrauma ${ }^{5}$. Prolonged mechanical ventilation (longer than two days) can lead to diaphragmatic atrophy and contractile dysfunction ${ }^{6}$. Ventilator-associated pneumonia is by far the most serious complication of mechanical ventilation, and is often due to increased number of day of mechanical ventilation and the intubation procedure itself?

The direct complications may lead to indirect complications such as increased hospital length of stay (LOS), emotional distress, increased costs, decreased bed availability and increase in patient morbidity and mortality ${ }^{8}$. The daily cost in ICU is estimated to be six fold higher than that of normal wards?. Again, longer ICU stays is associated with increased cost significantly, mostly due to laboratory, pharmacy and imaging charges $^{10}$. Thus, to avoid a lot of complication, cost and resource, patient should be weaned from mechanical ventilation at the earliest possible time. Considering the above mentioned facts that there was no exact data about this type of study in our country before. Therefore, this present study was undertaken to study efficacy of rapid shallow breathing index as a predictor of weaning of patients with prolonged mechanical ventilation by comparing between high and low rapid shallow breathing indexes.

\section{Methodology}

This prospective cohort study was conducted in the Department of Anesthesia, Pain Palliative \& Intensive
Care Unit of Dhaka Medical College Hospital, Dhaka, Bangladesh from January 2014 to December 2015 for a period of two (02) years. Patients on mechanical ventilation more than 48 hours with the age of 18 to 60 years were included in this study. Patients with tracheotomy, patients with spinal cord injury, self-extubation or unplanned extubation, patients who expired before spontaneous breathing trial, patients shifted to another hospital before weaning and within 48 hours of weaning were excluded from this study. Ethical clearance certificate from Ethical Review Committee of Dhaka Medical College was obtained. Standard weaning criteria was considered as resolution of the primary cause of respiratory failure, state of alertness, cooperation, response to commands and Glasgow coma scale (GCS) scores $\geq 9$. One type of ventilator (eVent Medical) was used in all patients. Primary and daily setting of ventilators and the decision to weaning of the patient was made by the ICU consultants. The arterial blood gases (ABG) values was checked during the weaning process and the patients was separated from mechanical ventilation by gradually decreasing the respiratory rate and pressure support (PS) in SIMV (synchronized intermittent mandatory ventilation) and PSV (pressure support ventilation) modes. Then spontaneous breathing trial was induced while the patient was attached to the ventilator with a low level of PS $\left(7 \mathrm{~cm}\right.$ of $\left.\mathrm{H}_{2} \mathrm{O}\right)$ and low PEEP $(5 \mathrm{~cm}$ of $\mathrm{H}_{2} \mathrm{O}$ or less). After one hour of spontaneous breathing trial (SBT) respiratory frequency (f) and exhaled tidal volume (EVT) in one minute was recorded from the ventilator scales. These data was served to calculate the minute volume and average tidal volume by dividing the minute volume by the respiratory frequency. RSBI was then measured through respiratory frequency divided by average exhaled tidal volume in liters (f/EVT). Throughout the weaning trial, the $\mathrm{FiO}_{2}$ setting was variable while vital signs, pulse oximetry, oxygen saturation $\left(\mathrm{SPO}_{2}\right)$ and hemodynamic status monitoring. After measuring RSBI, patients was separated from mechanical ventilator and given T-piece trial (1 to 4 hours) and finally extubated as per advice of ICU consultant and observed for 48 hours. If any patient was failed to T-piece trial then reconnected with mechanical ventilator and prepared the patient for further weaning. The patients were divided in two groups low RSBI $\leq$ 105 breath $/ \mathrm{min} / \mathrm{L}$ and high RSBI $>105$ breath $/ \mathrm{min} / \mathrm{L}$. These patients were prospectively followed up to 48 hours in ICU and HDU. Those groups of patients who were not reintubated within 48 hours are considered as success and those who needed reintubation or expired 
within 48 hours was considered as failure. Reintubation criteria within 48 hours of extubation was oxygen saturation below $90 \%$ in spite of high flow $\mathrm{O}_{2}$, respiratory rate $<8$ breath $/ \mathrm{min}$ or $>35$ breath $/$ min more than 5 minutes, pulse $>140$ beats $/ \mathrm{min}$, systolic $\mathrm{BP}>180$ $\mathrm{mm}$ of $\mathrm{Hg}$ or $40 \mathrm{~mm} \mathrm{Hg}$ above baseline or $<90 \mathrm{~mm}$ of $\mathrm{Hg}$, use of the accessory muscles of respiration, paradoxical breathing, reduced level of consciousness (GCS 8 or less), absence gag or cough reflex, bronchospasm, aspiration of lung secretions, excessive lung secretions, and further advice of consultants. Statistical analyses were carried out by using the Statistical Package for Social Sciences version 20.0 for Windows (SPSS Inc., Chicago, Illinois, USA). The mean values were calculated for continuous variables. The qualitative observations were expressed by frequencies and percentages.

\section{Results}

A total of 117 patients were included in this study. Majority 43(36.8\%) patients belonged to age 21 to 30 years and only $13(11.1 \%)$ patients belonged to age 50 to 60 years. The mean age was found $35.42 \pm 13.66$ years with range from 18 to 60 years (Table 1).

Table 1: Distribution of the Patients by Age $(n=117)$

\begin{tabular}{lcc}
\hline Age Group & Frequency & Percent \\
\hline$<30$ Years & 58 & 49.6 \\
31 to 50 Years & 46 & 29.9 \\
41 to 50 Years & 22 & 18.8 \\
$>50$ Years & 13 & 11.1 \\
Mean \pm SD & \multicolumn{3}{c}{$35.42 \pm 13.66$} \\
Range (min-max) & \multicolumn{3}{c}{18 to 60} \\
\hline
\end{tabular}

High RSBI was found in 25 cases of which reintubation was performed in $6(24.0 \%)$ cases and the rest $19(76.0 \%)$ cases were without reintubation required. Low RSBI was found in 92 cases of which reintubation was performed in 5(5.4\%) cases and the rest $87(94.6 \%)$ cases were without reintubation required. Thus, in RSBI evaluation of trail failure, true positive 6 cases, false positive 19 cases, false negative 5 cases and true negative 87 cases (Table 2).

Table 2: RSBI Findings in the Evaluation for Trail Failure $(\mathrm{n}=117)$

\begin{tabular}{|c|c|c|c|}
\hline \multirow[t]{2}{*}{ RSBI } & \multicolumn{2}{|c|}{ Re-intubation required (failure) } & \multirow[t]{2}{*}{ Percent } \\
\hline & Positive & Negative & \\
\hline High RSBI (>105) & $6(24.0 \%)$ & $19(76.0 \%)$ & $25(100.0 \%)$ \\
\hline Low RSBI $(\leq 105)$ & $5(5.4 \%)$ & $87(94.6 \%)$ & $92(100.0 \%)$ \\
\hline Total & 11 & 106 & 117 \\
\hline
\end{tabular}

The validity of RSBI evaluation for trail failure was correlated by calculating sensitivity, specificity, accuracy, positive and negative predictive values. The sensitivity of RSBI was low which was $54.5 \%$ (95\% CI $23.38 \%$ to $83.25 \%$ ). The specificity was high which was $82.1 \%$ (95\% CI $73.43 \%$ to $88.85 \%$ ). However, the Positive predictive value and Negative predictive value were $24.0 \%$ (95\% CI $13.84 \%$ to $38.30 \%$ ) and $94.6 \%$ (95\% CI $90.05 \%$ to $97.10 \%)$ respectively. The accuracy was found $79.5 \%(95 \%$ CI $71.03 \%$ to $86.39 \%$ ) (Table 3).

Table 3: Values of RSBI Evaluation for Prediction of Trail Failure

\begin{tabular}{lcc}
\hline Validity test & Value & 95\% CI \\
\hline Sensitivity & $54.5 \%$ & $23.38 \%$ to $83.25 \%$ \\
Specificity & $82.1 \%$ & $73.43 \%$ to $88.85 \%$ \\
Positive predictive value & $24.0 \%$ & $13.84 \%$ to $38.30 \%$ \\
Negative predictive value & $94.6 \%$ & $90.05 \%$ to $97.10 \%$ \\
Accuracy & $79.5 \%$ & $71.03 \%$ to $86.39 \%$ \\
\hline
\end{tabular}

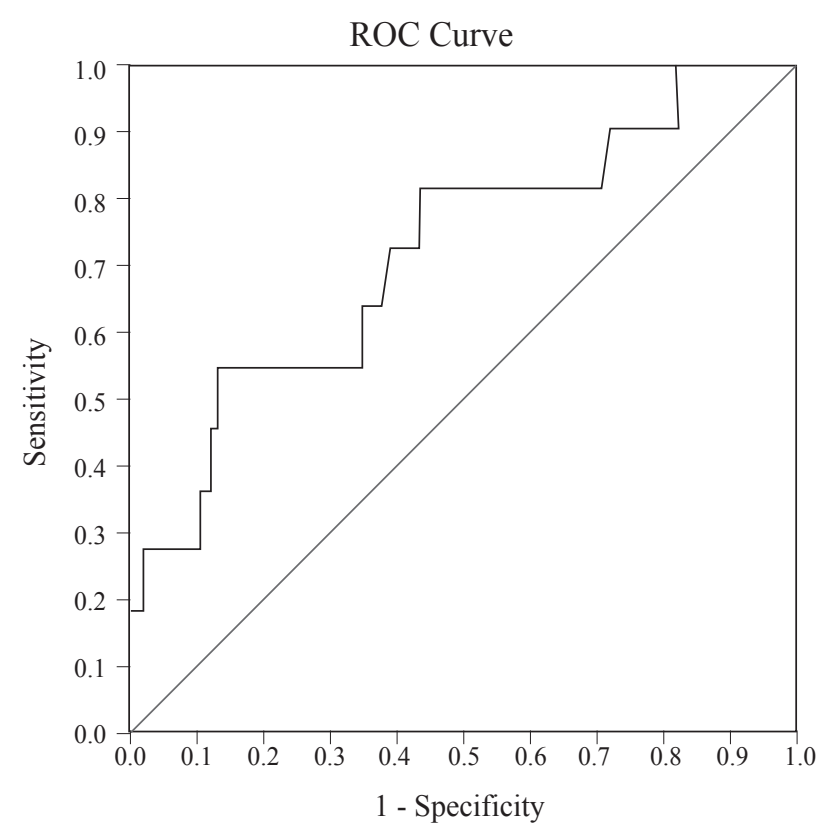

Figure I: Receiver-operator characteristic curves of the rapid shallow breathing index.

Receiver-operator characteristic (ROC) curve of RSBI for prediction of trial failure: The area under the receiver-operator characteristic (ROC) curves for the predictor of trail failure is depicted in the following table. Based on the receiver-operator characteristic (ROC) curves RSBI had the best area under curve (Figure I).

Receiver-operator characteristic (ROC) were constructed using RSBI of the weaning outcome, which 
Table 4: Receiver-operator characteristic (ROC) curve of RSBI for prediction of trail failure

\begin{tabular}{|c|c|c|c|c|c|c|c|c|c|c|}
\hline & \multirow[t]{2}{*}{$\begin{array}{l}\text { Cut of } \\
\text { value }\end{array}$} & \multirow[t]{2}{*}{ Sensitivity } & \multirow[t]{2}{*}{ Specificity } & \multirow[t]{2}{*}{ Accuracy } & \multirow[t]{2}{*}{ PPV } & \multirow[t]{2}{*}{ NPV } & \multirow[t]{2}{*}{ P value } & \multirow[t]{2}{*}{$\begin{array}{l}\text { Area under the } \\
\text { ROC curve }\end{array}$} & \multicolumn{2}{|c|}{$\begin{array}{l}\text { 95\% Confidence } \\
\text { interval (CI) }\end{array}$} \\
\hline & & & & & & & & & Lower & Upper \\
\hline RSBI (breath/min/L) & $\geq 88$ & 72.7 & 61.3 & 60.7 & 15.7 & 95.5 & $0.042^{\mathrm{s}}$ & 0.720 & 0.552 & 0.888 \\
\hline RSBI (breath/min/L) & $\geq 100$ & 54.5 & 77.4 & 72.6 & 18.2 & 94.0 & $0.041^{\mathrm{s}}$ & 0.720 & 0.552 & 0.888 \\
\hline RSBI (breath/min/L) & $\geq 105$ & 54.5 & 82.1 & 79.5 & 24.0 & 94.6 & $0.011^{\mathrm{s}}$ & 0.720 & 0.552 & 0.888 \\
\hline RSBI (breath/min/L) & $\geq 109$ & 27.3 & 89.6 & 83.6 & 21.4 & 92.2 & $0.126^{\mathrm{ns}}$ & 0.720 & 0.552 & 0.888 \\
\hline
\end{tabular}

gave a RSBI cut off value of $\geq 88$ as the value with a best combination of sensitivity $(72.7 \%)$ and specificity $(61.3 \%)$, accuracy $(60.7 \%)$, positive predictive value $(15.7 \%)$, negative predictive value $(95.5 \%)$ for trail failure. Using a threshold value of $\geq 100$, the sensitivity, specificity, accuracy, PPV, NPV for weaning outcome were $54.5 \%, 77.4 \%, 72.6 \%, 18.2 \%$ and $94.0 \%$ respectively. Using a threshold value of $\geq$ 105 , the sensitivity $(54.5 \%)$, specificity $(82.1 \%)$, accuracy $(79.5 \%)$, PPV (24.0\%), and NPV (94.6\%) for weaning outcome. Using a threshold value $\geq 109$, the sensitivity, specificity, accuracy, PPV and NPV were $27.3 \%, 89.6 \%, 83.6 \%, 21.4 \%$ and $92.2 \%$ for weaning outcome respectively (Table 4 ).

\section{Discussion}

Many ICUs use RSBI for weaning ${ }^{11}$, however, some studies have considered RSBI as a useless method ${ }^{12}$. Patel and his colleagues ${ }^{13}$ tried different methods for RSBI measurement. This prospective cohort study was carried out with an aim to investigate the efficacy of low rapid shallow breathing index on successful extubation and to assess the efficacy of high rapid shallow breathing index on successful extubation. A total of 117 patients of ICU who fulfilled the criteria of extubation after 48 hours in mechanical ventilation under Anesthesia, Pain, Palliative and Intensive Care unit of Dhaka Medical College Hospital, Dhaka were included in this study.

In this present study it was observed that $36.8 \%$ patients were in 3 rd decade and only $11.1 \%$ patient were in $6^{\text {th }}$ decade. The mean age was found $35.42 \pm 13.66$ years with range from 18 to 60 years. Goncalves et $\mathrm{a}^{14}$ and Berg et $\mathrm{a} \mathrm{l}^{15}$ showed the mean ages were $61.47 \pm 14.54$ years and $70 \pm 16$ years respectively. In another study Fadaii et al16 found that the mean age was $69.4 \pm 13.1$ years varying from 40 to 91 years, which all are higher with the current study. Similarly, higher mean age was also observed by Bien et $\mathrm{al}^{17}$, Patel et $\mathrm{al}^{13}$ and Chao and Scheinhorn ${ }^{18}$. The higher mean ages and age ranges obtained by the above authors due to geographical variations, racial, ethnic differences, genetic causes, different lifestyles, and increased life expectancy may have significant influence of their study patients.

In this study high RSBI group ( $>105$ breath $/ \mathrm{min} / \mathrm{L}$ ) failure is $6(24.0 \%)$ cases and success is $19(76.0 \%)$ cases. Low RSBI group $(\leq 105$ breath $/ \mathrm{min} / \mathrm{L})$ failure is found $5(5.4 \%)$ and success is $87(94.6 \%)$. Failure is significantly $(\mathrm{p}<0.05)$ higher in patients with high RSBI group. Fadaii et a ${ }^{16}$ have showed $90.0 \%$ patients had RSBI $\leq 105$ (breath $/ \mathrm{min} / \mathrm{L}$ ), among them $77.0 . \%$ patients had successful weaning and did not need reintubation while the remaining had unsuccessful weaning $(\mathrm{P}<0.05)$. The mean weaning index for patients with successful extubation is $66 \pm 57.2$ and $76.9 \pm 28.1$ for patients with unsuccessful extubaion, which is higher in unsuccessful extubaion but the difference is not significant between the means $(\mathrm{P}>0.05)$. Mahoori et $\mathrm{al}^{19}$ have showed the mean RSBI values which are significantly different between the failure and success groups. There was no significant difference regarding the values of other prediction criteria between the two groups. Kuo et $\mathrm{a}^{20}$ reported that RSBI was significantly higher in patients with extubation failure $(95.9 \pm 20.6)$ and trial failure $(98.0 \pm$ $50.0)$ than in patients with weaning success $(64.6 \pm$ $26.3)(\mathrm{p}<0.05)$. The above findings are consistent with the current study.

Bien et $\mathrm{al}^{17}$ used a threshold value of the RSBI of $\leq 105$ breaths $/ \mathrm{min} / 1$ to analyze the accuracies of predicting weaning success. The RSBI measured under each strategy had high sensitivity and positive predictive values and low specificity and negative predictive values as reported previously ${ }^{21}$. In Bien et $\mathrm{al}^{17}$ analysis of the area under the ROC curve showed that the predictive accuracies of the RSBI measured under these 5 strategies were lower than those reported previously ${ }^{22}$. Similarly, using a cutoff RSBI value of 105 breaths $/ \mathrm{min} / 1$, the diagnostic accuracies of predicting weaning success measured under these 5 strategies were also lower than those reported 
previously ${ }^{17,21}$. A heterogeneous population with a high percentage of acutely exacerbated COPD patients in Bien et $\mathrm{al}^{16}$ study patients may have contributed to these results ${ }^{22}$.

In RSBI evaluation of trail failure, true positive 6 cases, false positive 19 cases, false negative 5 cases and true negative 87 cases. The validity of RSBI evaluation for trail failure was correlated by calculating sensitivity $54.8 \%$, specificity $82.1 \%$, accuracy $79.5 \%$, positive predictive values $24.0 \%$ and negative predictive values $94.6 \%$. The sensitivity, specificity, PPV, and NPV of RSBI compared favorably to those of Sassoon and Mahutte ${ }^{23}$ and $\mathrm{Tu}^{24}$ studies that used more complicated parameters to predict weaning success. As noted in previous studies, the prevalence of weaning failure can influence the performance of a weaning index. The prevalence of weaning failure in turn can be influenced by various factors that differ among institutions, including clinical judgment, criteria for selecting patients for weaning trials and also for defining respiratory failure.

Receiver-operator characteristic (ROC) were constructed using RSBI of the weaning outcome it was observed in this study that, RSBI cut off value $\geq 88$ had best combination of sensitivity $72.7 \%$ and specificity $61.3 \%$, accuracy $60.7 \%$, positive predictive value $15.7 \%$, negative predictive value $95.5 \%$ for trail failure. Using a threshold value of $\geq 100$, the sensitivity $54.5 \%$, specificity $77.4 \%$, accuracy $72.6 \%$, PPV $18.2 \%$ and NPV $94.0 \%$ for weaning outcome of trail failure. Using a threshold value of $\geq 105$, the sensitivity $54.5 \%$, specificity $82.1 \%$, accuracy $79.5 \%$, PPV $24.0 \%$ and NPV 94.6\% for weaning outcome of trail failure. Using a threshold value $\geq 109$, the sensitivity $27.3 \%$, specificity $89.6 \%$, accuracy $83.6 \%$, PPV $21.4 \%$ and NPV $92.2 \%$ for weaning outcome of trail failure. Kuo et al20 observed by using a threshold value of 105, the sensitivity $91.0 \%$, specificity $25.0 \%$ and accuracy $85.0 \%$ for weaning outcome. The best accuracy of RSBI $75.0 \%$ was achieved when a threshold value of 88 was used, with a sensitivity $83.0 \%$, specificity $64.0 \%$, positive predictive value $78.0 \%$ and negative predictive value $70.0 \%$, which is closely resembled with the present study. Sensitivity, specificity, and predictive values are of limited value for predicting outcomes in weaning studies because they generally apply to single cut-off points obtained by another researcher ${ }^{17}$. A threshold value of 100 was used, the accuracy of RSBI in Kuo et $\mathrm{al}^{20}$ study $84.0 \%$ was similar to that of RSBI reported by Chatila et $\mathrm{a}^{25}$ measured at 30 minutes after an SBT $85.0 \%$.
Chao et $\mathrm{al}^{26}$ found that the predictive accuracy of Yang and Tobin's ICU RSBI threshold of 105 was only $59.0 \%$. In Chao and Scheinhorn ${ }^{18}$ RT-implemented weaning protocol the authors chose a low RSBI threshold of 80 for advancing the patient to 1-hour SBT. This conservative threshold, chosen to minimize false positives (patients below threshold RSBI but who fail to tolerate 1-hour SBT), was from a published evidence-based recalculation of Yang and Tobin's data for the ICU population ${ }^{28}$. Chao and Scheinhorn ${ }^{18}$ found that this threshold value allowed $28.0 \%$ of patients to start SBTs earlier, and $89.0 \%$ of these patients tolerated the $\mathrm{SBT}^{27}$. RSBI correlated well in Chao and Scheinhorn ${ }^{27}$ study with the patients' ability to tolerate 1-hour SBT (area under the receiver operating characteristic curve 0.844). They calculated that an RSBI threshold of 80 had a sensitivity of $62.4 \%$ and specificity of $88.5 \%$. The authors found that by re-plotting the sensitivity and specificity separately against the $\log$ of RSBI they were able to calibrate a threshold RSBI to the desired combination of sensitivity and specificity. Increasing the RSBI threshold to 97 increased the sensitivity by $15.0 \%$ while sacrificing specificity by $4.0 \%$.

\section{Conclusion}

In conclusion the rapid shallow breathing index (RSBI) is a useful index for prediction of weaning failure and success of patients with prolonged mechanical ventilation and it should be worthy to note here that RSBI help the physician in the rational approach of patient management. Furthermore, RSBI has definite value in the diagnosis of weaning outcome and can be regarded as a sensitive and specific index for prediction of weaning success and failure of patients with prolonged mechanical ventilation. Further studies may be undertaken on RSBI regarding successful weaning from mechanical ventilation including large number of patients.

\section{References}

1. American Thoracic Society, Infectious Diseases Society of America: Guidelines for the management of adults with hospital-acquired, ventilator-associated, and healthcare-associated pneumonia. Am J Respir Crit Care Med 2005;171:388-16

2. Eskandar, N, Apostolakos MJ. Weaning from mechanical ventilation. Crit Care Clin 2007;23:263-74

3 . Hasani A, Grbolar A. Principles of weaning from the mechanical ventilation, AIM 2008;16(2):83-85

4. Marelich GP, Murin S, Battistella F, Inciardi J, Vierra T, Roby M. Protocol Weaning of Mechanical Ventilation in Medical and Surgical Patients by RespiratoryCare Practitioners and Nurses: Effect on Weaning Time and Incidence of Ventilator-Associated Pneumonia. Chest. 2000;118(2):459-67 
5. Beckmann U, Gillies DM. Factors associated with reintubation in intensive care: an analysis of causes and outcomes. Chest. 2001;120(2):538-42

6. Powers SK, Kavazis AN, Levine S. Prolonged mechanical ventilation alters diaphragmatic structure and function. Critical care medicine. 2009;37(10 Suppl):S347-53

7. Vincent JL, Bihari DJ, Suter PM, Bruining HA, White J, Nicolas-Chanoin MH, Wolff M, Spencer RC, Hemmer M. The prevalence of nosocomial infection in intensive care units in Europe: results of the European Prevalence of Infection in Intensive Care (EPIC) Study. JAMA. 1995;274(8):639-44

8. Fagon JY, Chastre J, Vuagnat A, Trouillet JL, Novara A, Gibert C. Nosocomial pneumonia and mortality among patients in intensive care units. JAMA 1996;275(11):866-9

9. Miwa K, Mitsuoka M, Takamori S, Hayashi A, Shirouzu K. Continuous monitoring of oxygen consumption in patients undergoing weaning from mechanical ventilation. Respiration. 2003;70(6):623-30.

10. Krishnan JA, Moore D, Robeson C, Rand CS, Fessler HE. A prospective, controlled trial of a protocol-based strategy to discontinue mechanical ventilation. American journal of respiratory and critical care medicine. 2004;169(6):673-8

11. Shikora SA, Benotti PN, Johannigman JA. The oxygen cost of breathing may predict weaning from mechanical ventilation better than the respiratory rate to tidal volume ratio. Archives of Surgery. 1994;129(3):269-74

12. Hess DR. Mechanical ventilation strategies: what's new and what's worth keeping? Respiratory care. 2002;47(9):1007-17

13. Patel KN, Ganatra KD, Bates JH, Young MP. Variation in the rapid shallow breathing index associated with common measurement techniques and conditions. Respiratory Care. 2009;54(11):1462-6

14. Gonçalves EC, Silva EC, Basile Filho A, Auxiliadora-Martins M, Nicolini EA, Gastaldi AC. Low pressure support changes the rapid shallow breathing index (RSBI) in critically ill patients on mechanical ventilation. Brazilian Journal of Physical Therapy 2012;16(5):368-74.

15. Berg KM, Lang GR, Salciccioli JD, Bak E, Cocchi MN, Gautam S, Donnino MW. The rapid shallow breathing index as a predictor of failure of noninvasive ventilation for patients with acute respiratory failure. Respiratory care. 2012;57(10):1548-54.

16. Fadaii A, Amini SS, Bagheri B, Taherkhanchi B. Assessment of rapid shallow breathing index as a predictor for weaning in respiratory care unit. Tanaffos. 2012;11(3):28-31

17. Bien MY, Lin YS, Shie HG, Yang YL, Shih CH, Wang JH,
Cheng KC. Rapid shallow breathing index and its predictive accuracy measured under five different ventilatory strategies in the same patient group. Chin J Physiol. 2010 Feb 28;53(1):1-0

18. Chao DC, Scheinhorn DJ. Determining the best threshold of rapid shallow breathing index in a therapist-implemented patient-specific weaning protocol. Respiratory care. 2007;52(2):159-65

19. Mahoori AR, Nowruzinia S, Farasatkish R, Ali Mollasadeghi G, Kianfar AA, Toutounchi MZ. Assessment of the rapid shallow breathing index as a predictor of weaning of patients with prolonged mechanical ventilation. Tanaffos. 2007;6(3):30-5.

20. Kuo PH, Kuo SH, Yang PC, Wu HD, Lu BY, Chen MT. Predictive value of rapid shallow breathing index measured at initiation and termination of a 2-hour spontaneous breathing trial for weaning outcome in ICU patients. Journal of the Formosan Medical Association. 2006;105(5):390-8.

21. Conti G, Montini L, Pennisi MA, Cavaliere F, Arcangeli A, Bocci MG, Proietti R, Antonelli M. A prospective, blinded evaluation of indexes proposed to predict weaning from mechanical ventilation. Intensive care medicine. 200430(5):830-6 22. Cohen JD, Shapiro M, Grozovski E, Singer P. Automatic tube compensation-assisted respiratory rate to tidal volume ratio improves the prediction of weaning outcome. Chest 2002;122(3):980-4

23. Sassoon CS, Kees Mahutte C. Airway occlusion pressure and breathing pattern as predictors of weaning outcome. American Review of Respiratory Disease. 1993;148:860-66

24. Tu X. Application of multi-predictors in the ventilator weaning process. Chinese Journal Of Tuberculosis And Respiratory Diseases 2004;27(12):829-32

25. Chatila W, Jacob B, Guaglionone D, Manthous CA. The unassisted respiratory rate-tidal volume ratio accurately predicts weaning outcome. The American journal of medicine. 1996;101(1):61-7

26. Chao DC, Scheinhorn DJ, Hassenpflug M, Artinian B, Catlin J. Rapid shallow breathing index (RSBI) in prolonged ventilator dependency. Chest. 1995;108:190S

27. Scheinhorn DJ, Chao DC, Stearn-Hassenpflug M, Wallace WA. Outcomes in post-ICU mechanical ventilation: a therapist-implemented weaning protocol. Chest. 2001;119(1):236-42.

28. Jaeschke RZ, Meade MO, Guyatt GH, Keenan SP, Cook DJ. How to use diagnostic test articles in the intensive care unit: diagnosing weanability using f/Vt. Critical care medicine. 1997;25(9):1514-21 